\title{
Putting Stars in the Gap: in situ Irradiation and Heating of Synthetic SiC and Implications for the Origins of C-rich Circumstellar Materials
}

\author{
TJ Zega ${ }^{1,2 *}$, J Bernal $^{3}$, JY Howe ${ }^{4}$, P Haenecour ${ }^{1}$, S Amari ${ }^{5}$, and LM Ziurys ${ }^{3,6}$ \\ 1. Lunar and Planetary Laboratory, University of Arizona, Tucson, AZ, USA. \\ 2. Dept. of Materials Science and Engineering, University of Arizona, Tucson, AZ, USA. \\ 3. Dept. of Chemistry and Biochemistry, University of Arizona, Tucson, AZ, USA. \\ 4. Dept. of Materials Science and Engineering, and Dept. of Chemical Engineering and Applied \\ Chemistry, University of Toronto, Ontario, Canada. \\ 5. Physics Dept. and McDonnell Center for the Space Sciences, Washington University, St. Louis, MO, \\ USA. \\ 6. Dept. of Astronomy, University of Arizona, Tucson, AZ, USA. \\ * Corresponding author: tzega@lpl.arizona.edu
}

Primitive meteorites contain within them the preserved ashes of ancient stars. These 'presolar' grains condensed in gaseous circumstellar environments and made their way through the interstellar medium to our local part of the galaxy where the solar system was forming some 4.56 billion years ago. Graphite and $\mathrm{SiC}$ are among the largest $(\leq 30 \mu \mathrm{m})$ types of presolar materials and thus, also the most well studied [1]. $\mathrm{SiC}$, in particular, was shown to occur mostly in the 3C polytype, reflecting the thermodynamic conditions in which it formed [2]. Moreover, grains of $\mathrm{SiC}$ and other metal-carbides were observed inside of graphite [3], raising questions regarding the sequence in which these materials condensed in their host circumstellar envelopes. To better understand such materials, and moreover, how reduced $\mathrm{C}$ can form in what is known from astronomical observations [4] to be an otherwise H-rich environment (along with other abundant elements such as $\mathrm{O}, \mathrm{N}$, and $\mathrm{S}$ ), we report here results on in situ heating and irradiation experiments inside the TEM with synthetic SiC.

A 3C polytype of $\mathrm{SiC}$ (99\% purity) was dropcasted onto $\mathrm{SiN}$ support films as part of the Norcada microelectromechanical systems (MEMS) chip used for in situ heating, which we have previously demonstrated [5,6]. The MEMS chips were loaded into a Hitachi 'Blaze' heating holder and together loaded into the Hitachi H9000 transmission electron microscope (TEM) located at the intermediate voltage electron microscope tandem facility at Argonne National Laboratory. We heated the SiC grains at 5 ${ }^{\circ} \mathrm{C} / \mathrm{min}$ to $1000{ }^{\circ} \mathrm{C}$, and then held it isothermally and irradiated it with $\mathrm{Xe}$ at $150 \mathrm{keV}$ to 15 displacements per atom (dpa) over approximately two hours. After heating and irradiation, the sample was analyzed at the University of Arizona using a Hitachi HF5000 aberration-corrected scanning TEM (S/TEM). The HF5000 is equipped with energy-dispersive X-ray and electron energy-loss spectrometers (EDS/EELS, respectively) for chemical analysis. The imaging analysis was carried out at $200 \mathrm{keV}$, whereas EELS analyses were performed at the $\mathrm{C}, \mathrm{K}$ edge at $60 \mathrm{keV}$.

Figure 1 shows one of the grid locations that was monitored in the experiment. Particles are mostly monodispersed, but localized areas contain clumps of several particles. High-resolution (phase-contrast) TEM (HRTEM) imaging shows that the bulk of the interior of the large $(\geq 100 \mathrm{~nm})$ particles contains the original 3C SiC structure. In comparison, smaller $(<100 \mathrm{~nm})$ particles show breakdown of the 3C structure on their edges. HRTEM reveals lattice fringes containing interplanar $d$-spacings of $0.34 \mathrm{~nm}$, which correspond to the (002) spacing for graphite, occur on the edges of most grains. In addition, localized parts of the edges contain complete breakdown of long-range order, whereas others contain small (1 nm) 
circular structures that are similar in size and shape to $\mathrm{C}_{60}$. EELS spectrum imaging on several areas of one of the particles confirms the HRTEM observations. Filtered maps, constructed from the EELS spectrum images, show clearly the localized distribution of $s p^{2}$-hybridized (planar trigonal) graphitic bonding on the edge of the grain and $s p^{3}$-hydribidzed (tetrahedral) $\mathrm{SiC}$ bonding in the core.

The experimental data we report here provide new insight into the circumstellar origins of $\mathrm{SiC}$ and graphite. The $\mathrm{SiC}$ remnant core and graphite shell produced in our in situ experiments are consistent with the previous observations of bonafide circumstellar materials [3]. During the in situ heating and irradiation, $\mathrm{Si}$ atoms were knocked out of the $\mathrm{SiC}$ lattice. $\mathrm{C}$ was left behind where it reconstructed to form graphene, graphite, and structures that are similar in size and shape to $\mathrm{C}_{60}$. In other words, our in situ experimental data suggest that thermodynamic conditions simply need to favor SiC condensation followed by heating and irradiation to form reduced $\mathrm{C}$ materials in circumstellar environments. The mechanism we propose here can explain the previous microstructural observations of these circumstellar materials without the need for high pressures or supernovae environments [7].

\section{References:}

[1] T Bernatowicz et al., in "Meteorites and the Early Solar System II", ed. DS Lauretta, HY McSween Jr., (The University of Arizona Press, Tucson) p. 109.

[2] T Daulton et al., Science 296, (2002), p. 1852.

[3] TK Croat et al., The Astrophysical Journal 139 (2010), p. 2159.

[4] Y Zhang and S Kwok, The Astrophysical Journal 730 (20101, p. 126.

[5] Thompson et al., Meteoritics \& Planetary Science 52 (2017), p. 413.

[6] Keller LP et al., Lunar and Planetary Science Conference (2018) Abstract \#2594

[7] Research supported by NASA grant NNX15AJ22G (T.J.Z.), NSF grant AST-1515568 (L.M.Z.), and NASA Agreement No. NNX15AD94G. T.J.Z. acknowledges NASA (grants \#NNX12AL47G and \#NNX15AJ22G) and NSF (grant \#1531243) for funding the instrumentation in the Kuiper Materials Imaging and Characterization Facility, UA.
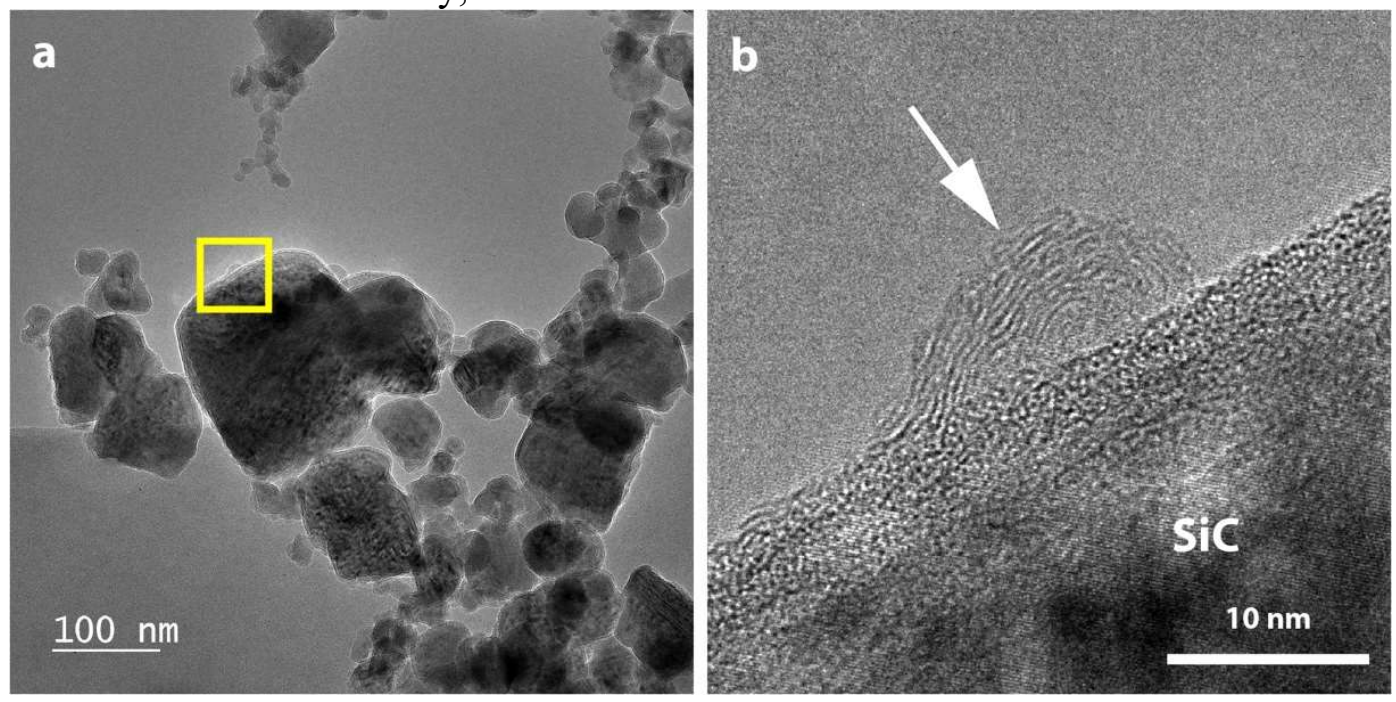

Figure 1. TEM images of synthetic SiC and graphite. (a) SiC particles dispersed on MEMS support. (b) HRTEM image of graphite (white arrow) formed on edge of SiC. 